\title{
Sistemas de Información Geográfica y Localización de un Relleno Sanitario en Cerro de Pasco
}

\author{
Geographic Information Systems and Location of a Sanitary Landfill in Cerro \\ de Pasco
}

\author{
Jesús Manuel Estacio Vidal', Oscar Rafael Tinoco Gómez², Jenny Díaz Tafur, \\ Rosa Karol Moore Torres ${ }^{4}$
}

Recibido: 12/02/2021 - Aprobado: 01/10/2021 - Publicado: 23/12/2021

\begin{abstract}
RESUMEN
El presente estudio surge a raíz de la problemática en la ubicación de infraestructuras de disposición final (relleno sanitario) de residuos sólidos donde se toma en consideración diversos criterios de análisis siguiendo lo dispuesto en el Reglamento del DL N 1278 Ley de Gestión Integral de Residuos Sólidos DS N 014-2017-MINAM. Se realizó el análisis de localización óptima utilizando los procesos de Análisis Espacial Multicriterio (AEMC) así como el proceso de análisis jerárquico (AHP) aplicándolo en un Sistema de Información Geográfica (ArcGis). La investigación tuvo como propósito localizar las áreas adecuadas para la disposición final de residuos sólidos en la ciudad de Cerro de Pasco. Los resultados presentan la consideración de 6 alternativas de localización para el relleno sanitario, todas ellas comprendidas entre los 3 y $4.13 \mathrm{~km}$ de distancia de la ciudad, bajo criterios físicos, ambientales, socioeconómicos y legales. Se concluye que los sistemas de información geográfica, el análisis espacial multicriterio y el análisis jerárquico son apropiados para la resolución de problemas de localización.
\end{abstract}

Palabras claves: Sistemas de información geográfica; arctoolbox; análisis espacial multicriterio; rellenos sanitarios; residuos sólidos.

\begin{abstract}
This study arises as a result of the problem in the location of solid waste final disposal infrastructures (Landfill) where various analysis criteria are taken into consideration following the provisions of DL Regulation N 1278 Comprehensive Waste Management Law Solid DS N 014-2017-MINAM. The optimal location analysis was carried out using the Multicriteria Spatial Analysis (AEMC) processes as well as the hierarchical analysis process (AHP) applying it in a Geographic Information System (ArcGis). The purpose of the investigation was to locate the appropriate areas for the final disposal of solid waste in the city of Cerro de Pasco. The results present the consideration of 6 alternative locations for the sanitary landfill, all of them between 3 and $4.13 \mathrm{~km}$ away from the city, under physical, environmental, socioeconomic and legal criteria. It is concluded that geographic information systems, multi-criteria spatial analysis and hierarchical analysis are appropriate for solving location problems.
\end{abstract}

Keywords: Geographic information systems; arctoolbox; multicriteria spatial analysis; landfills; solid waste.

1 Universidad Nacional Mayor de San Marcos, Lima, Perú. Ingeniero Geólogo con Posgrado en Ciencias Ambientales con mención en Desarrollo Sustentable en Minería y Recursos Energéticos.

Autor para correspondencia: jestacio24@gmail.com - ORCID: https://orcid.org/0000-0002-8323-6582

2 Universidad Nacional Mayor de San Marcos, Facultad de Ingeniería Geológica, Minera, Metalúrgica y Geográfica, Asesor de tesis. Lima, Perú. Docente. E-mail: otinocog@unmsm.edu.pe - ORCID: https://orcid.org/0000-0002-7927-931X

3 Universidad San Antonio Abad de Cusco. Bióloga con Posgrado en Ciencias, Mención Ecología y Recursos Naturales. Cusco, Perú. E-mail: ienny.dita@gmail.com - ORCID: https:/lorcid.org/0000-0002-6431-7459

4 Universidad Nacional Mayor de San Marcos, Facultad de Ingeniería Industrial, Unidad de Posgrado, Lima, Perú. Docente. E-mail: kmooret@unmsm.edu.pe - ORCID: https://orcid.org/0000-0002-7608-9377 


\section{INTRODUCCIÓN}

Desde su creación las ciudades han generado desechos sólidos finales que debían ser depositados en lugares apropiados, en la actualidad tanto el aumento demográfico como la urbanización han impactado en la gestión de dichos residuos especialmente a lo relacionado con la determinación de una ubicación óptima para la disposición de los mismos, siendo en años recientes un problema de alta preocupación (Mohammed, y otros, 2019); (Gordillo, 2019); (Palacios, 2018); (Bahrani, Ebadi, Ehsani, Yousefi, \& Maknoon , 2016) ya que se requiere una detallada evaluación de factores tanto ambientales, económicos como sociales (Ding, Zhu, Wu, \& Zuo, 2020); (Nguyen, Chou, Fang, Hoang, \& Nguyen, 2020). En cuanto a los factores ambientales destacan la distancia a las superficies de agua, zonas de conservación de agua, reservas naturales. aeropuertos, tierras especiales, terrenos agrícolas, pendientes de relieve y elevación, orientación de los vientos, permeabilidad del suelo (Deswal \& Laura, 2018); (Ding, Zhu, Wu, \& Zuo, 2020), cabe resaltar que la importancia relativa de estos factores difiere según la ubicación geográfica (Sisay, Legesse, \& Getahun, 2020); (Mohammed, y otros, 2019); (Çeliker, Yıldız, \& Nacar, 2019). Con respecto a los factores económicos destacan las pendientes, asentamientos, tierras de bajo valor económico como tierras libres y matorrales, suelos, redes viales, áreas protegidas, aeropuertos, pozos, distancia a los ríos y riachuelos (Sisay, Legesse, \& Getahun, 2020) y en relación a los factores sociales destacan la distancia de los caminos, distancia de las áreas pobladas, planes de uso y coberturas de tierras (Deswal \& Laura, 2018). Muchos de estos factores no son de naturaleza cuantitativa pasando a ser denominados atributos (Saaty, 1977) por lo que no son sujetos de comparación matemática, así mismo no todos los factores son valorados bajo el mismo grado de importancia por lo que se les asigna un peso a cada factor relativo entre sí.

En el ámbito latinoamericano, específicamente en la ciudad de Macas, Colombia, (Palacios, 2018) abordó la localización de un relleno sanitario aplicando el proceso de jerarquía analítica (AHP), técnica que conlleva a una comparación pareada de factores (la pendiente, permeabilidad del suelo, vías, ríos, usos del suelo) entre sí, obteniéndose un peso relativo para cada factor. Así mismo (Chida, 2020) desarrolló la localización de un relleno sanitario en el Cantón Tena, Ecuador, mediante una combinación del ArcMap 10.5 y el AHP, utilizando para ello diferentes criterios, destacando "distancia a vías, distancia a los asentamientos humanos, distancia a los recursos hídricos, pendiente, permeabilidad, susceptibilidad de inundación, uso de suelo, la precipitación y la profundidad del nivel freático".

Los Sistemas de Información Geográfica (GIS) han evolucionado como una técnica importante para el análisis de idoneidad del uso del suelo. Los GIS tiene la capacidad reconocer, correlacionar y analizar el espacio con relación a los datos mapeados, así como establecer enlaces entre diversas fuentes de información. (Espejo, 2017) aplicó sistemas de información geográfica y AHP para la localización de un relleno sanitario en Chachapoyas, en su estudio destaca la importancia del procesamiento de datos raster como un instrumento para el análisis de riesgos, minimización de las problemáticas medioambientales y permitir la toma de mejores decisiones geoambientales.

\section{MÉTODOS}

\section{1. Área de Estudio}

El departamento de Pasco está ubicado en la zona centro del Perú, el cual está constituido por tres provincias: Daniel Alcides Carrión, Oxapampa y Pasco, contando esta última con 56959 habitantes los cuales representan el $22.1 \%$ de la población del departamento y por lo tanto mostrando su relación directa entre la densidad poblacional y la generación de los residuos sólidos urbanos. Así mismo el departamento de Pasco cuenta con cuatro rellenos sanitarios, donde los distritos beneficiarios son: Oxapampa, Chontabamba, Huancabamba, Pozuzo, Ninacaca y Tinyahuarco (Ministerio del Ambiente, 2021) así como 25 áreas degradadas o también llamados botaderos (Organismo de Evaluación y Fiscalización Ambiental, 2021). Según el Inventario Nacional de Áreas Degradadas por Residuos Sólidos Municipales elaborado por el Organismo de Evaluación y Fiscalización Ambiental se identificaron 1 637 áreas bajo estas condiciones en el año 2021.

Según las estadísticas ambientales (Dossiers) elaborado por el Ministerio del Ambiente en el año 2020, el departamento de Pasco generó 37 mil toneladas al año de residuos sólidos municipales, en tanto que en la provincia de Pasco existe una generación municipal anual de residuos de 19144.56 toneladas al año, una generación municipal diaria de 52.45 toneladas por día y una generación municipal per cápita de $0.48 \mathrm{~kg} / \mathrm{hab} / \mathrm{d}$ áa (Ministerio del Ambiente, 2020) (Tabla 1).

\subsection{Materiales}

\subsubsection{Imágenes satelitales}

Se usaron las más actualizadas de SENTINEL-2 obtenida del geoservidor EOS Data Analytics.

Tabla 1. Ciclo de la Gestión Integral de los residuos sólidos

\begin{tabular}{cccccc}
\hline $\mathbf{N}^{\circ}$ & Provincia & $\mathbf{N}^{\circ}$ Habitantes & $\begin{array}{c}\text { Generación Municipal Anual } \\
\text { (t/año) }\end{array}$ & $\begin{array}{c}\text { Generación Municipal Diaria } \\
\text { (t/día) }\end{array}$ & $\begin{array}{c}\text { Generación Municipal Per } \\
\text { Cápita (kg/hab/día) }\end{array}$ \\
\hline 1 & Daniel Alcides Carrión & 123,015 & $3,986.50$ & 10.92 & 0.49 \\
2 & Oxapampa & 43,580 & $14,329.81$ & 39.26 & 0.76 \\
3 & Pasco & 87,470 & $19,144.56$ & 52.45 & 0.48 \\
& Total & 254,065 & 37460.87 & 102.63 & 0.56 \\
\hline
\end{tabular}

Nota. Información obtenida del Ministerio del Ambiente - Dirección General de Gestión de Residuos Sólidos, 2020. 


\subsubsection{Información geoespacial}

Se utilizó la información técnica necesaria para el procesamiento cartográfico que fueron obtenidas de geoservidores de libre acceso (Tabla 2).

\subsection{Metodología}

La elaboración de la base de datos geoespacial se realizó siguiendo el esquema de trabajo que se muestra en la Figura 1.

\subsubsection{Análisis espacial multicriterio}

El análisis espacial multicriterio es un conjunto de técnicas donde se establecen objetivos y alternativas de solución que son comparadas bajo distintos criterios, los cuales son modelados según los pesos asignados por los expertos con el fin de seleccionar la alternativa óptima (Posada, 2015). Cabe destacar que es práctica habitual en la aplicación de los métodos del análisis espacial multicriterio el considerar un número pequeño de alternativas sobre una cantidad limitada de criterios (Barredo \& Gomez, 2005).

2.3.2 Análisis Espacial Multicriterio y la selección de sitios óptimos para infraestructura de disposición final de residuos sólidos

Herramienta desarrollada en la década del 70 y aplicada en diversos estudios relacionados en áreas como economía, salud, política, planificación urbana, etc. El proceso de análisis jerárquico es una comparación de pares basada en los juicios de expertos para obtener las escalas de prioridades,

Tabla 2. Materiales cartográficos

\begin{tabular}{ccc}
\hline $\mathbf{N}^{\circ}$ & Insumo & Fuente \\
\hline 1 & Imagen SENTINEL-2 & EOS Data Analytics \\
2 & Limite Político Administrativo & Instituto Nacional de Estadística e Informática \\
3 & Red Vial & Ministerio de Transportes y Comunicaciones \\
4 & Centros Poblados & Instituto Nacional de Estadística e Informática \\
5 & Topografía (Curvas de Nivel) & Instituto Geográfico Nacional \\
6 & Hidrografía & Instituto Geográfico Nacional \\
7 & Aeródromos & Ministerio de Transportes y Comunicaciones \\
8 & Fallas Geológicas & Instituto Geológico Minero, Metalúrgico \\
9 & Geología & Instituto Geológico Minero, Metalúrgico \\
10 & Peligros Geológicos & Instituto Geológico Minero, Metalúrgico \\
11 & Áreas Naturales Protegidas & Servicio Nacional de Áreas Naturales Protegidas por el Estado \\
12 & Precipitación & Servicio Nacional de Meteorología e Hidrología / World Clim \\
13 & Sitios Arqueológicos & Ministerio de Cultura \\
14 & Cobertura Vegetal & Ministerio del Ambiente \\
15 & Granjas avícolas, porcinas, etc. & Servicio Nacional de Sanidad Agraria del Perú \\
16 & Pasivos Mineros & Ministerio de Energía y Minas \\
\hline
\end{tabular}

Nota. Tabla elaborada por Estacio Jesús para fines de la investigación.

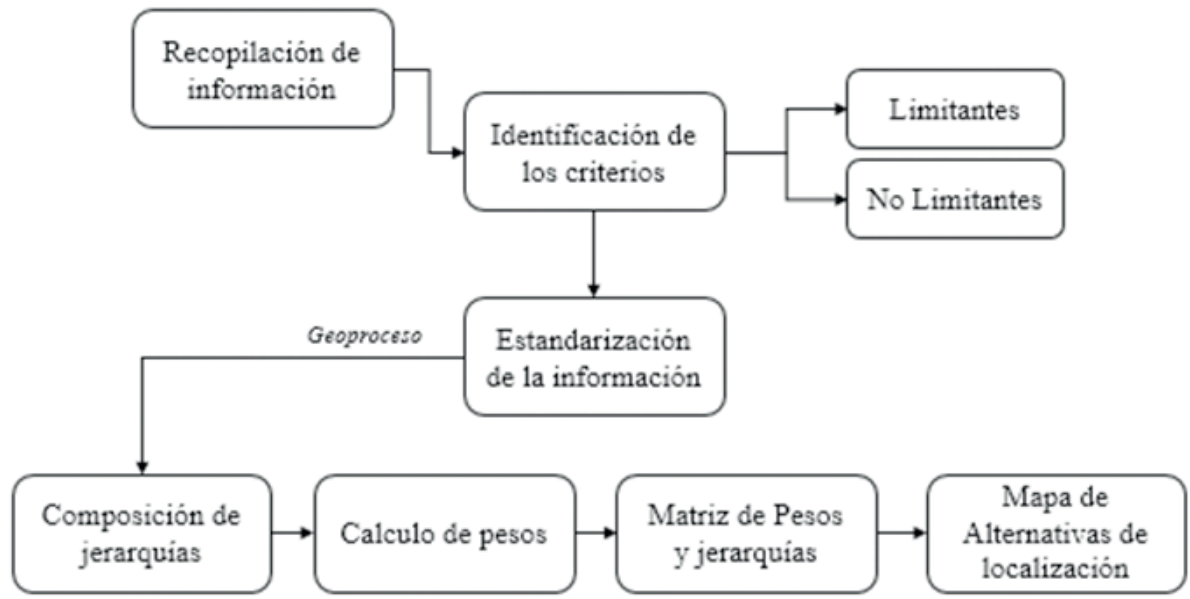

Figura 1. Esquema metodológico de la investigación.

Nota. Esquema elaborado considerando la metodología para el desarrollo del Análisis Jerárquico (AHP), según lo establecido por (Saaty, 1977) 
esta metodología está basada en la descomposición, juicio comparativo y simplificación de las importancias de los problemas de decisión (Saaty, 1977). El principio de juicio comparativo se expresa a través de la comparación por pares de los elementos de cada nivel de jerarquía. La simplificación de las importancias supone tomar cada una de las escalas de prioridades obtenidas en cada nivel de jerarquía y elaborar un conjunto de prioridades globales para el último nivel de la jerarquía, que será utilizado para evaluar las distintas alternativas (Barredo \& Gomez, 2005).

a. Recopilación de la Información: es el enfoque sistemático de reunir información geoespacial necesaria para realizar el proceso.

b. Identificación de los criterios: se definen los criterios que serán parte del modelo de procesos además de establecer los insumos necesarios.

c. Estandarización de la información: se realiza una normalización de la información como unificar el sistema de coordenadas geográficas.

d. Composición de jerarquías: se realiza la construcción de matrices en base a los juicios de expertos para obtener las escalas de prioridades.

e. Cálculo de pesos: ya teniendo los criterios escalados jerárquicamente, se realiza el cálculo de los pesos donde se realiza una prueba de consistencia para determinar si la matriz de pesos y jerarquías es la adecuada.

f. Matriz de Pesos y jerarquías: es el resultado obtenido de los procesos anteriores donde se tiene en limpio los criterios y subcriterios con sus respectivos pesos y jerarquías.

g. Mapa de Alternativas de localización: al aplicar el modelo con la matriz de pesos y jerarquías a través del geoproceso de superposición ponderada en los sistemas de información geográfica se obtiene un mapa con las alternativas de sitios de infraestructura de disposición final de residuos sólidos.

El proceso de la gestión integral de los residuos sólidos se inicia desde la generación hasta la disposición final de residuos con el fin de prevenir los riesgos a la salud de la población y afectación al medio ambiente. La realidad es que se cuenta con limitadas infraestructuras para la disposición final de los residuos sólidos municipales y es por ello por lo que se generan los botaderos o áreas degradadas por residuos sólidos. En la Guía para el Diseño y Construcción de Infraestructuras para disposición final de Residuos Sólidos Municipales se establecen 21 criterios de selección (Ministerio del Ambiente, 2018), tomando en consideración los criterios estipulados en los artículos 109, 110 y 111 del (Ministerio del Ambiente, 2017).

Teniendo como herramienta principal los Sistemas de Información Geográfica (SIG) se puede combinar con la metodología del Análisis Espacial Multicriterio (AEMC) para dar solución a la problemática de ubicación de infraestructura de disposición final de residuos sólidos. Para la realización de operaciones especiales durante el análisis se elaboró una base de datos geoespacial que contenía datos elementales y datos temáticos. Con lo anterior mencionado se obtuvo un mapa de factibilidad espacial que contribuyó a la solución de la problemática referida a la ubicación de lugares óptimos para la construcción de infraestructura de disposición final de residuos sólidos en la ciudad de Pasco.

\section{RESULTADOS}

\subsection{Diagnóstico}

Hacia el noroeste de la ciudad de Pasco, capital de la provincia de Pasco, se encuentra el botadero de desmonte denominado Rumiallana que debido al crecimiento de la población genera impactos tanto sociales como ambientales. Este botadero se encuentra sobre el depósito de desmonte de la mina de propiedad de la Empresa Administradora Cerro S.A.C. (Figura 2).

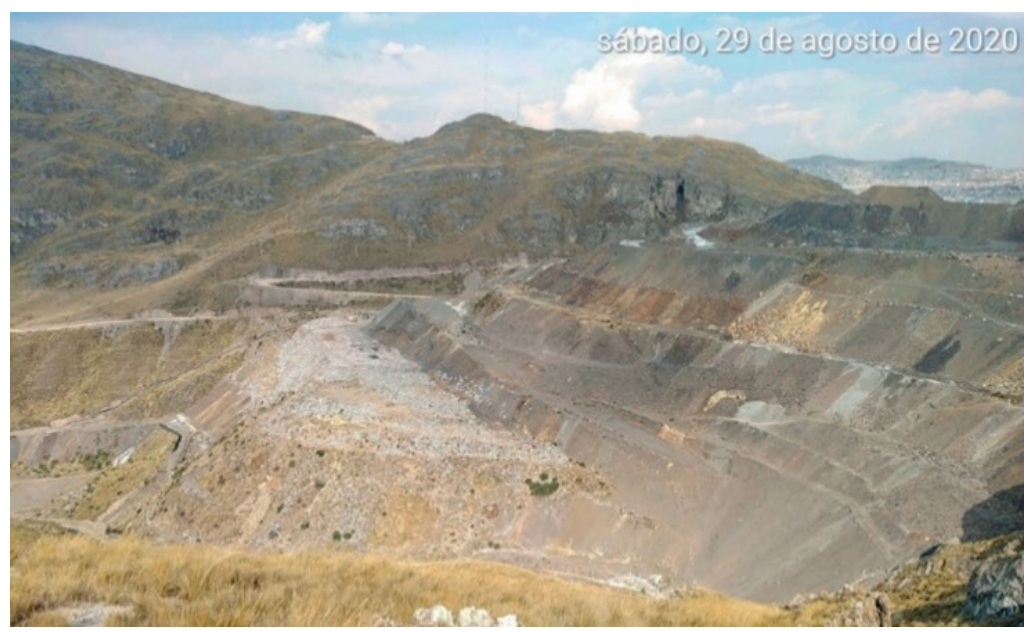

Figura 2. Botadero Rumiallana, Cerro de Pasco.

Nota. Fotografía de la vista panorámica del botadero de Rumiallana, Cerro de Pasco - Perú. 
En el año 2018, la Contraloría General de la Republica verificó la pérdida de estabilidad y el riesgo de posible deslizamiento del depósito de desmonte Rumiallana debido a la sobrecarga de los residuos sólidos provenientes de los distritos de Chaupimarca, Yanacancha y Simón Bolívar (Contraloría General de la República, 2018) lo que conllevó a que en junio de ese mismo año se emita la Resolución Ministerial 232-2018-MINAM declarándose en emergencia la gestión y manejos de los residuos sólidos en los distritos de Chaupimarca, Yanacancha y Simón Bolívar.

\subsection{Identificación de los criterios}

Al momento de analizar los criterios de ubicación de una infraestructura de disposición final de residuos sólidos se consideró lo estipulado en los articulos $\mathrm{N}^{\circ} 109,110$ y 111 del Decreto Supremo No 014-2017-MINAM (Ministerio del Ambiente, 2017) (Tabla 3).

\subsection{Estandarización de la información}

Para la normalización de la información se necesitó convertir los archivos digitales registrados en el formato shapefile (.shp) a formato raster; así como la unificación del sistema de proyección a WGS 84 Zona 18 Sur que es donde se encuentra el área del presente estudio. Estas acciones de estandarización se realizaron con las herramientas de ArcToolbox "Polygon to Raster" y "Project" que se encuentran en el sistema de información geográfica ArcMap.

\subsection{Composición de jerarquías}

Para realizar la composición de jerarquías se utilizó el proceso de análisis jerárquico (AHP) desarrollado por (Saaty, 1977), donde se construyó una matriz de comparación de pares. En esta matriz se comparan cada criterio de las columnas con las filas teniendo en consideración el valor de importancia. La comparación entre criterios iguales tendrá un valor de importancia igual a 1 y cuando se comparen criterios diferentes se tiene que fijar cuál de los criterios es considerado el más importante utilizando la escala de Saaty.

Ya elaborada la matriz de comparación de pares sigue sumar los valores de cada columna, luego se divide cada elemento de la matriz por el total que da la suma de cada columna. Para comprobar que la matriz sea consistente o sólida debe ser menor a 0.1 y para eso se realiza el cálculo de índice de consistencia (IC), índice aleatorio (RI) y la razón de consistencia (RC). Para hallar el valor de RC se requiere calcular el valor de $\lambda$ max:

$$
\lambda \max =\mathbf{V} * \mathbf{B}
$$

$\lambda \max =$ máximo valor propio de la matriz.

$\mathrm{V}=$ vector priorización o peso de cada variable con respecto a los demás criterios.

$\mathrm{B}=$ vector suma ponderada, que es la sumatoria de elementos de cada columna de la matriz.

Teniendo el valor de $\lambda \max$, se determina el valor de Índice de consistencia que indica el grado de desviación de los pesos de los criterios analizados respecto al grado de importancia de cada uno de los criterios, utilizando la siguiente formula:

$$
\mathrm{IC}=(\lambda \max -\mathbf{n}) /(\mathbf{n}-\mathbf{1})
$$

$\mathrm{n}=$ número de criterios analizados

Por último, se aplica la siguiente fórmula para determinar la relación de consistencia:

$$
\mathbf{R C}=\mathbf{I C} / \mathbf{R I}
$$

$\mathrm{RI}=$ Índice aleatorio, existe un cuadro del valor de RI para matrices de orden 1 hasta 15 (Saaty, 1977). Nota:

\begin{tabular}{|c|c|c|c|c|}
\hline Ámbito & $\mathbf{N}^{\circ}$ & Criterios & Tipo & Limitantes \\
\hline \multirow{5}{*}{ Físico } & 1 & Permeabilidad & $A$ & \multirow{5}{*}{$>500 \mathrm{~m}$} \\
\hline & 2 & Pendiente & A & \\
\hline & 3 & Distancia euclidiana a Fallas Geológicas ${ }^{* *}$ & B & \\
\hline & 4 & Distancia euclidiana a Peligros Geológicos* & B & \\
\hline & 5 & Precipitación & A & \\
\hline \multirow{3}{*}{ Ambiental } & 6 & Distancia euclidiana a Recursos hídricos* & B & \multirow[t]{3}{*}{$>500 \mathrm{~m}$} \\
\hline & 7 & Cobertura Vegetal $^{*}$ & A & \\
\hline & 8 & Distancia euclidiana a Área Natural Protegida & B & \\
\hline \multirow{6}{*}{ Socio-económico } & 9 & Distancia euclidiana a Zonas Urbanas* & B & \multirow[t]{2}{*}{$>500 \mathrm{~m}$} \\
\hline & 10 & Distancia euclidiana a vías & B & \\
\hline & 11 & Distancia euclidiana a granjas* & B & \multirow[t]{3}{*}{$>500 \mathrm{~m}$} \\
\hline & 12 & Distancia euclidiana a Sitios Arqueológicos & B & \\
\hline & 13 & Distancia euclidiana a Pasivos Mineros & B & \\
\hline & 14 & Distancia euclidiana a Aeródromos* & B & $>13 \mathrm{~km}$ \\
\hline
\end{tabular}
Los Valores del Índice Aleatorio (RI) para los diferentes

Tabla 3. Criterios utilizados en el Análisis Espacial Multicriterio

Nota. ${ }^{*}$ Criterios restrictivos según DS N Decreto Supremo N 014-2017-MINAM. ${ }^{* *}$ Criterios restrictivos según RM N 459-2018-MINAM. 
"n", obtenidos mediante la simulación de 100,000 matrices (Moreno-Jiménez, Aguarón, \& Escobar, 2001).

De este procedimiento se consigue la matriz de comparación de pares con la relación de consistencia de 0.021 como se muestra en la Tabla 4.

\subsection{Cálculo de pesos}

A partir de la matriz de comparación de pares jerarquizada, se realizó el análisis espacial multicriterio a cada uno de los subcriterios dentro de los criterios utilizando la metodología antes utilizada, esto con el fin de obtener subcriterios con peso por jerarquías (Tabla 5).

\subsection{Adecuación de base de datos geoespacial}

Teniendo los pesos ponderados por cada criterio en la matriz se procedió a integrarlos y adecuarlos en los insumos cartográficos para lo cual se transformó cada shapefile del criterio tipo A en formato raster de la Tabla 3 utilizando la herramienta del ArcToolbox "Polygon to Raster". Los criterios tipo B se trabajaron con las distancias de alejamiento utilizando la herramienta "Euclidean distance". Para colocar los valores de los pesos ponderados de cada subcriterio se utilizó la herramienta "Reclassify" (Figura 3).

\subsection{Cálculo para determinar la localización óptima de relleno sanitario}

Con la información geoespacial desarrollada se procedió a calcular las áreas de localización optima dependiendo de su nivel de ubicación, para lo cual se utilizó la herramienta "Raster Calculator" y se procedió a sumar los valores de cada criterio multiplicando por cada peso que se le asignó.

$$
\mathrm{NL}=\boldsymbol{\Sigma} \mathbf{P} * \mathbf{C}
$$

$$
\begin{aligned}
& \text { NA }=\text { Nivel de Localización } \\
& \mathrm{P}=\text { Peso ponderado del criterio } \\
& \mathrm{C}=\text { Raster del criterio }
\end{aligned}
$$

El desarrollo de la fórmula conllevó a obtener un raster con la suma lineal ponderada de cada criterio analizado, que a su vez contenían 5 subcriterios obteniéndose los rangos en el nivel de localización presentado en la Tabla 6.

Como producto final del proceso desarrollado se obtuvo como resultado un mapa con aquellas áreas para

\begin{tabular}{|c|c|c|c|c|c|c|c|c|c|c|c|c|c|c|c|c|}
\hline Criterios & & 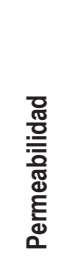 & 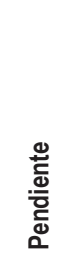 & 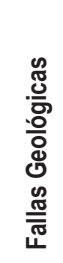 & 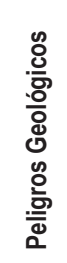 & 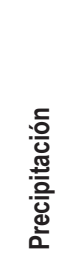 & 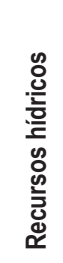 & 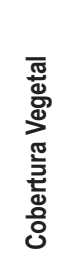 & 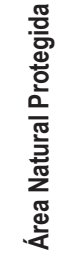 & 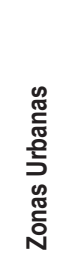 & 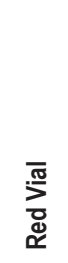 & 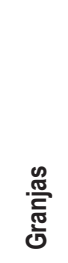 & 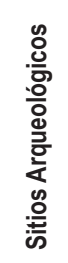 & 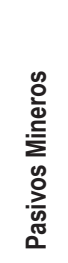 & 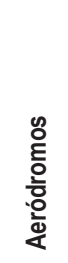 & $\begin{array}{l}\text { \& } \\
\text { d }\end{array}$ \\
\hline \multirow{5}{*}{ Físico } & Permeabilidad & 1.00 & 2.00 & 0.50 & 0.50 & 2.00 & 0.33 & 1.00 & 1.00 & 0.50 & 1.00 & 0.50 & 2.00 & 3.00 & 3.00 & 0.066 \\
\hline & Pendiente & 0.50 & 1.00 & 0.33 & 0.33 & 1.00 & 0.33 & 0.50 & 0.50 & 0.50 & 1.00 & 0.50 & 1.00 & 3.00 & 3.00 & 0.047 \\
\hline & Fallas Geológicas & 2.00 & 3.00 & 1.00 & 1.00 & 3.00 & 1.00 & 2.00 & 2.00 & 1.00 & 2.00 & 1.00 & 3.00 & 3.00 & 3.00 & 0.114 \\
\hline & Peligros Geológicos & 2.00 & 3.00 & 1.00 & 1.00 & 3.00 & 1.00 & 1.00 & 1.00 & 1.00 & 2.00 & 1.00 & 2.00 & 3.00 & 3.00 & 0.100 \\
\hline & Precipitación & 0.50 & 1.00 & 0.33 & 0.33 & 1.00 & 0.33 & 0.50 & 0.50 & 0.50 & 1.00 & 0.50 & 1.00 & 3.00 & 3.00 & 0.047 \\
\hline \multirow{3}{*}{ Ambiental } & Recursos hídricos & 3.00 & 3.00 & 1.00 & 1.00 & 3.00 & 1.00 & 3.00 & 3.00 & 1.00 & 3.00 & 1.00 & 3.00 & 3.00 & 3.00 & 0.132 \\
\hline & Cobertura Vegetal & 1.00 & 2.00 & 0.50 & 1.00 & 2.00 & 0.33 & 1.00 & 1.00 & 1.00 & 2.00 & 1.00 & 2.00 & 3.00 & 3.00 & 0.080 \\
\hline & Área Natural Protegida & 1.00 & 2.00 & 0.50 & 1.00 & 2.00 & 0.33 & 1.00 & 1.00 & 1.00 & 2.00 & 1.00 & 2.00 & 3.00 & 3.00 & 0.080 \\
\hline \multirow{6}{*}{$\begin{array}{l}\text { Socio } \\
\text { económico }\end{array}$} & Zonas Urbanas & 2.00 & 2.00 & 1.00 & 1.00 & 2.00 & 1.00 & 1.00 & 1.00 & 1.00 & 2.00 & 1.00 & 2.00 & 3.00 & 3.00 & 0.094 \\
\hline & Red Vial & 1.00 & 1.00 & 0.50 & 0.50 & 1.00 & 0.33 & 0.50 & 0.50 & 0.50 & 1.00 & 0.50 & 1.00 & 2.00 & 2.00 & 0.047 \\
\hline & Granjas & 2.00 & 2.00 & 1.00 & 1.00 & 2.00 & 1.00 & 1.00 & 1.00 & 1.00 & 2.00 & 1.00 & 2.00 & 3.00 & 3.00 & 0.094 \\
\hline & Sitios Arqueológicos & 0.50 & 1.00 & 0.33 & 0.50 & 1.00 & 0.33 & 0.50 & 0.50 & 0.50 & 1.00 & 0.50 & 1.00 & 3.00 & 3.00 & 0.048 \\
\hline & Pasivos Mineros & 0.33 & 0.33 & 0.33 & 0.33 & 0.33 & 0.33 & 0.33 & 0.33 & 0.33 & 0.50 & 0.33 & 0.33 & 1.00 & 1.00 & 0.026 \\
\hline & Aeródromos & 0.33 & 0.33 & 0.33 & 0.33 & 0.33 & 0.33 & 0.33 & 0.33 & 0.33 & 0.50 & 0.33 & 0.33 & 1.00 & 1.00 & 0.026 \\
\hline$n=14$ & $\lambda \max =14.435$ & & 0.0 & & & -1 & & & & & & & & & & \\
\hline
\end{tabular}
la ubicación de relleno sanitario tal como se presenta en Figura 4.

Tabla 4. Matriz de comparación de pares.

Nota. Matriz elaborada por Estacio J., Tinoco O., Díaz J., Moore R. para fines de la investigación 
Tabla 5. Matriz de pesos y jerarquías

\begin{tabular}{|c|c|c|c|c|c|c|c|c|}
\hline $\begin{array}{c}\text { Sub } \\
\text { Criterio }\end{array}$ & Descripción & Peso & $\begin{array}{c}\text { Sub } \\
\text { Criterio }\end{array}$ & Descripción & Peso & $\begin{array}{c}\text { Sub } \\
\text { Criterio }\end{array}$ & Descripción & Peso \\
\hline \multirow{5}{*}{ 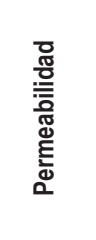 } & Permeabilidad Muy Baja & 0.468 & ஜ & $>4000 \mathrm{~m}$ & 0.350 & \multirow{5}{*}{ 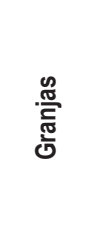 } & $>5000 \mathrm{~m}$ & 0.388 \\
\hline & Permeabilidad Baja & 0.281 & $\frac{\overline{\underline{T}}}{0}$ & $2000-4000 \mathrm{~m}$ & 0.263 & & $2000-5000 m$ & 0.291 \\
\hline & Permeabilidad Moderada & 0.170 & c) & $1000-2000 m$ & 0.203 & & $1000-2000 m$ & 0.187 \\
\hline & Permeabilidad Alta & 0.050 & 峞 & $500-1000 m$ & 0.157 & & $500-1000 m$ & 0.106 \\
\hline & Permeabilidad Muy Alta & 0.032 & 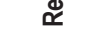 & $0-500 \mathrm{~m}^{*}$ & 0.026 & & $0-500 \mathrm{~m}^{*}$ & 0.029 \\
\hline \multirow{5}{*}{ 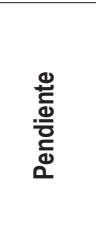 } & $10-15 \%$ & 0.464 & \multirow{5}{*}{ 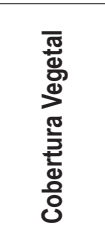 } & Pajonal & 0.441 & \multirow{5}{*}{ 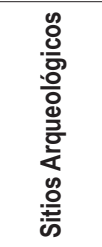 } & $>5000 \mathrm{~m}$ & 0.345 \\
\hline & $15-25 \%$ & 0.287 & & Matorral & 0.284 & & $2000-5000 m$ & 0.258 \\
\hline & $25-50 \%$ & 0.152 & & Agricultura andina & 0.191 & & $1000-2000 m$ & 0.222 \\
\hline & $0-10 \%$ & 0.067 & & Bosque ${ }^{*}$ & 0.054 & & $500-1000 m$ & 0.148 \\
\hline & $>50 \%$ & 0.030 & & Bofedal $^{*}$ & 0.029 & & $0-500 \mathrm{~m}$ & 0.026 \\
\hline \multirow{5}{*}{ 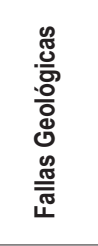 } & $>4000 \mathrm{~m}$ & 0.470 & \multirow{5}{*}{ 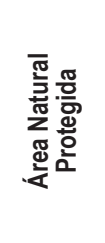 } & $>5000 \mathrm{~m}$ & 0.427 & \multirow{5}{*}{ 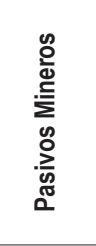 } & $>5000 \mathrm{~m}$ & 0.35 \\
\hline & $2000-4000 m$ & 0.270 & & $2000-5000 \mathrm{~m}$ & 0.294 & & $2000-5000 m$ & 0.263 \\
\hline & $1000-2000 m$ & 0.151 & & $1000-2000 m$ & 0.188 & & $1000-2000 m$ & 0.203 \\
\hline & $500-1000 m$ & 0.080 & & $500-1000 m$ & 0.062 & & $500-1000 m$ & 0.157 \\
\hline & $0-500 \mathrm{~m}^{*}$ & 0.028 & & $0-500 \mathrm{~m}^{*}$ & 0.029 & & $0-500 \mathrm{~m}$ & 0.026 \\
\hline \multirow{5}{*}{ 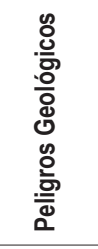 } & $>2000 \mathrm{~m}$ & 0.350 & \multirow{5}{*}{ 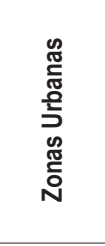 } & $>5000 \mathrm{~m}$ & 0.350 & \multirow{5}{*}{ 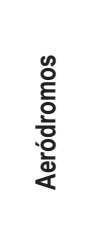 } & $>75 \mathrm{~km}$ & 0.279 \\
\hline & $1500-2000 \mathrm{~m}$ & 0.263 & & $2000-5000 \mathrm{~m}$ & 0.263 & & $50-75 \mathrm{~km}$ & 0.247 \\
\hline & $1000-1500 m$ & 0.203 & & $1000-2000 \mathrm{~m}$ & 0.203 & & $25-50 \mathrm{~km}$ & 0.235 \\
\hline & $500-1000 \mathrm{~m}$ & 0.157 & & $500-1000 m$ & 0.157 & & $13-25 \mathrm{~km}$ & 0.213 \\
\hline & $0-500 \mathrm{~m}^{*}$ & 0.026 & & $0-500 \mathrm{~m}^{*}$ & 0.026 & & $0-13 \mathrm{~km}^{*}$ & 0.026 \\
\hline \multirow{5}{*}{ 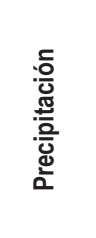 } & $0-250 m$ & 0.448 & \multirow{5}{*}{ 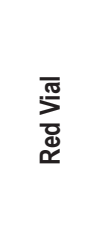 } & $>1500 \mathrm{~m}$ & 0.350 & & & \\
\hline & $250-500 \mathrm{~mm}$ & 0.291 & & $1000-1500 m$ & 0.263 & & & \\
\hline & $500-750 \mathrm{~mm}$ & 0.169 & & $600-1000 \mathrm{~m}$ & 0.203 & & & \\
\hline & $750-1000 \mathrm{~mm}$ & 0.061 & & $300-600 \mathrm{~m}$ & 0.157 & & & \\
\hline & $>1000 \mathrm{~mm}$ & 0.030 & & $0-300 \mathrm{~m}$ & 0.026 & & & \\
\hline
\end{tabular}

Nota. *Limite restrictivo según DS N Decreto Supremo N 014-2017-MINAM. Matriz elaborada por Estacio J., Tinoco O., Díaz J., Moore R. para fines de la investigación

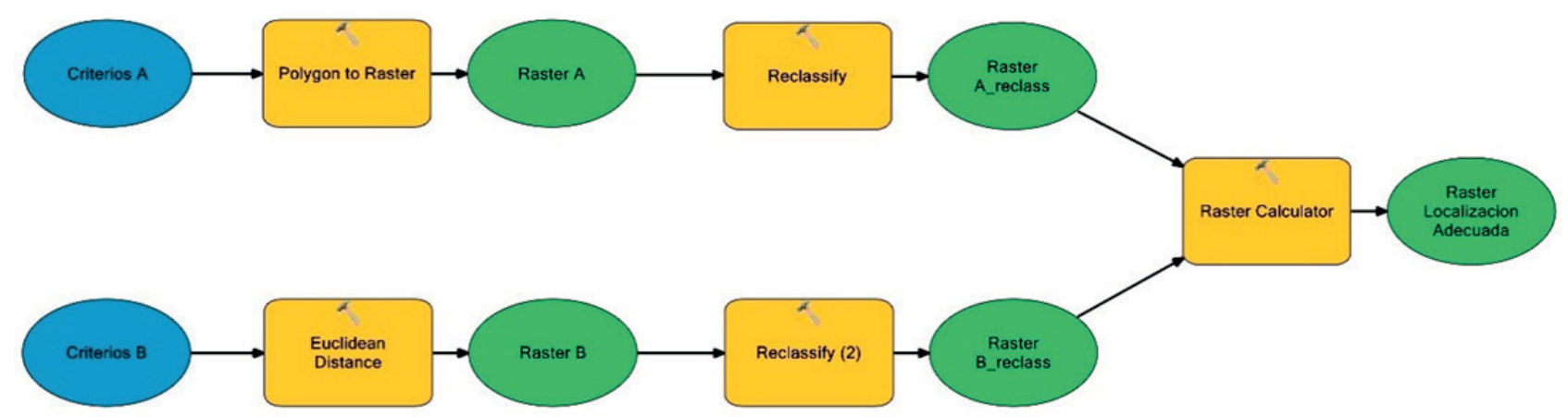

Figura 3. Proceso simplificado de adecuación de base de datos geoespacial.

Nota. Ilustración elaborada por Estacio J., Tinoco O., Díaz J., Moore R. para fines de la investigación.

Tabla 6. Nivel de Localización Adecuada de Relleno Sanitario

\begin{tabular}{cccc}
\hline & Rango & Localización Adecuada \\
\hline $0.274 \leq$ LA $<$ & 0.396 & OPTIMO \\
\hline 0.189 & $\leq$ LA $<0.274$ & ACEPTABLE \\
\hline $0.113 \leq$ LA $<0.189$ & INSUFICIENTE \\
\hline $0.028 \leq$ LA $<0.113$ & NO APTO \\
\hline
\end{tabular}

Nota. Información elaborada por Estacio J., Tinoco O., Díaz J., Moore R. para fines de la investigación 
Debido a que se trabajó en el área de toda la provincia, se obtuvieron muchas áreas con niveles óptimos y aceptables para la ubicación del Relleno Sanitario. Con el fin de delimitar un área de trabajo más pequeño se optó por buscar aquellas no mayores a los 5 kilómetros de la ciudad de Cerro de Pasco con el fin de reducir los gastos operativos referentes al traslado de los residuos sólidos, además se tomó en consideración la superficie mínima recomendada para relleno sanitario de 5 a 10 hectáreas según la Resolución Ministerial N 459-2018-MINAM. Con lo mencionado se presentaron seis áreas en la Tabla 7 así como su representación gráfica en la Figura 5.

\section{DISCUSIÓN}

Seleccionar el sitio de un relleno sanitario siguiendo las recomendaciones y las regulaciones de los organismos reguladores es un problema técnicamente desafiante, pero económicamente beneficioso, ya que garantiza una selección adecuada por primera vez evitando problemas ambientales y cambiando del sitio en una etapa posterior. El estudio ha asegurado que todos los factores y regulaciones se utilizaron con una combinación de técnicas como GIS y AHP.

La presente investigación se elaboró mediante el uso de las herramientas de los sistemas de información geográfica (GIS); tomando como insumos cartográficos información geoespacial de distintas instituciones del Estado, considerándose conveniente la aplicación del análisis espacial multicriterio y el proceso de análisis jerárquico, cuyos resultados fueron seis áreas óptimas que corroboran y concuerda con el estudio realizado por (Barredo \& Gomez, 2005).

Se tomó en consideración 14 criterios al momento de realizar el análisis espacial multicriterio los cuales concuerdan con los 21 criterios que establece la Guía para el Diseño y Construcción de Infraestructuras de Disposición Final de Residuos Sólidos Municipales RM N459-2018-MINAM (Ministerio del Ambiente, 2018), los criterios no considerados corresponden a los de evaluación complementaria especifica en aquellas áreas seleccionadas para localización de relleno sanitario como estudios

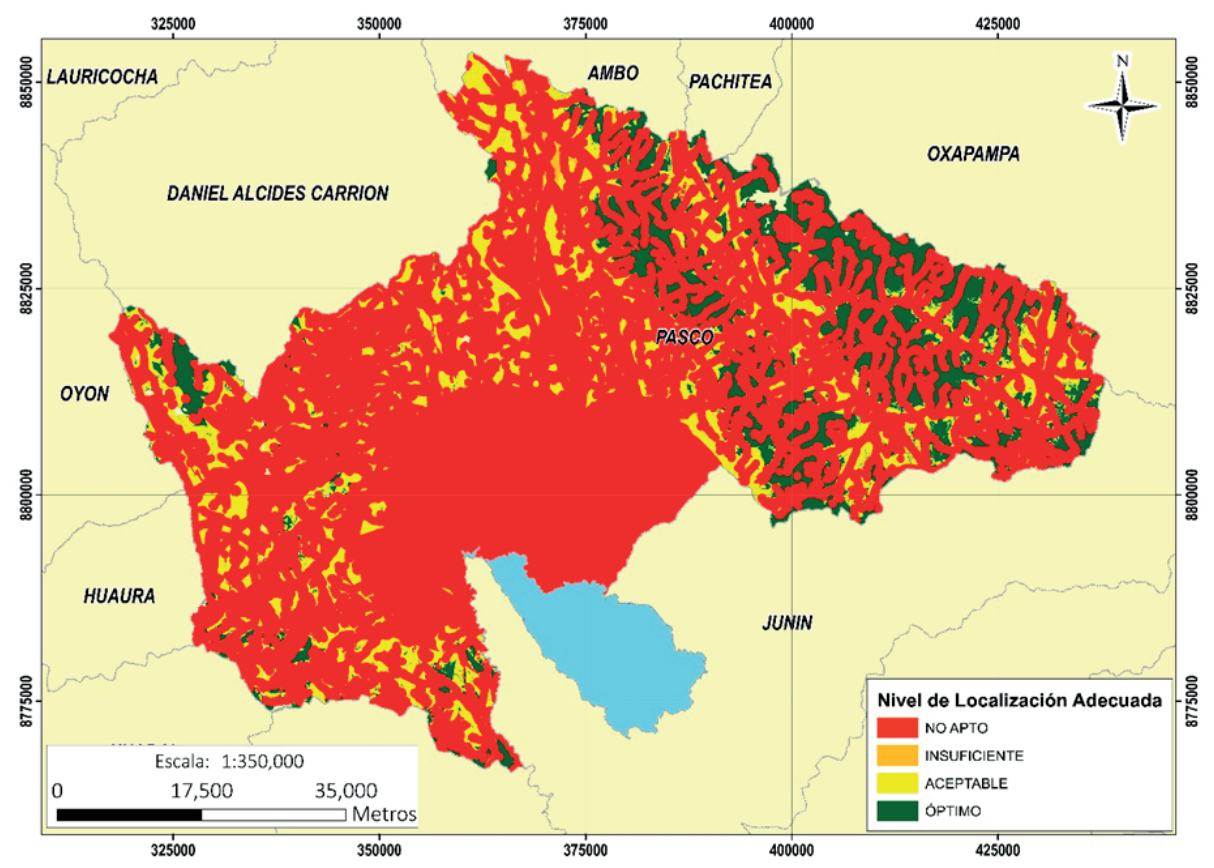

Figura 4. Mapa de áreas de localización adecuada.

Nota. Ilustración elaborada por Estacio Jesús para fines de la investigación

Tabla 7. Alternativas de localización de Relleno Sanitario

\begin{tabular}{cc}
\hline Alternativas & Distancia desde la ciudad \\
\hline 1 & 3.33 \\
2 & 3.50 \\
3 & 2.37 \\
4 & 3.30 \\
5 & 3.45 \\
6 & 4.13 \\
\hline
\end{tabular}

Nota. Información elaborada por Estacio J., Tinoco O., Díaz J., Moore R. para fines de la investigación 


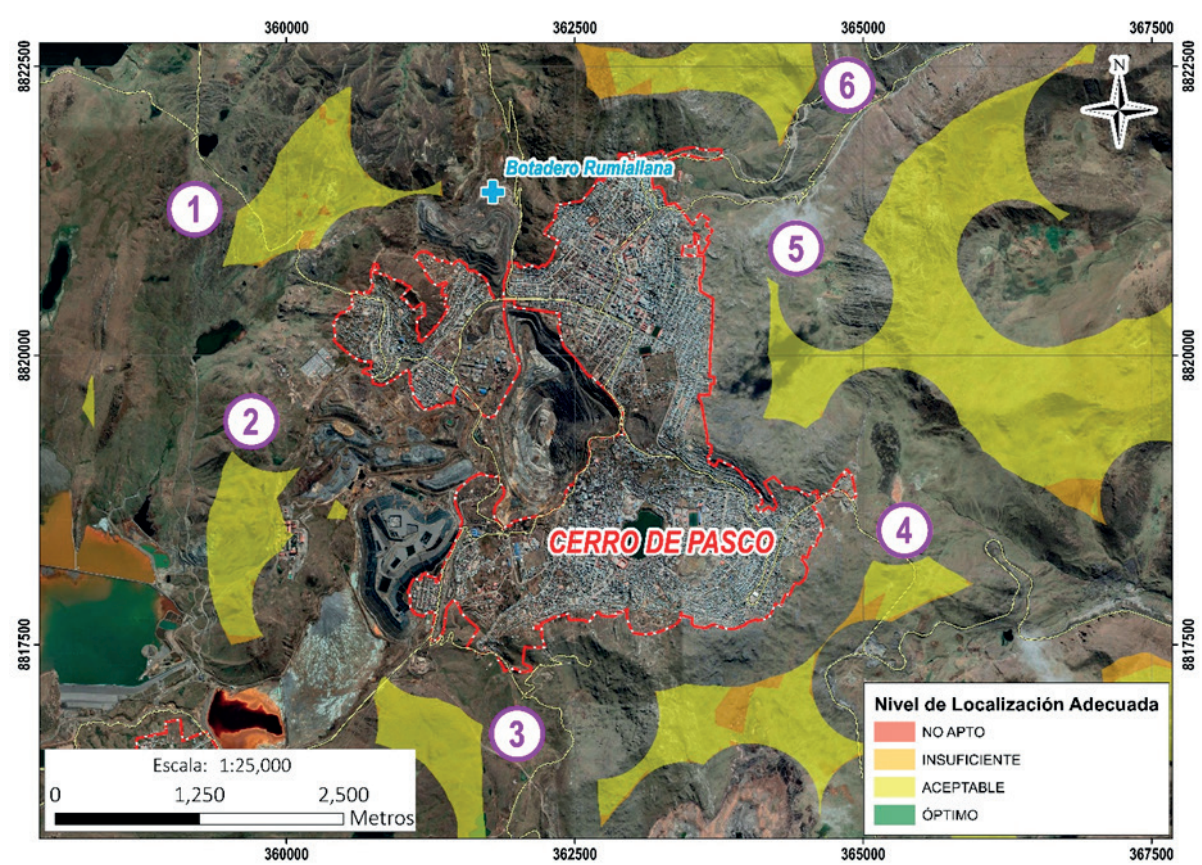

Figura 5. Mapa de áreas de localización cerca de la ciudad de Cerro de Pasco. Nota. llustración elaborada por Estacio Jesús para fines de la investigación

geofísicos, estudios geotécnicos, información de estación meteorológico para determinar la velocidad y dirección del viento, y opinión pública de la población.

Los criterios que coinciden con los estudios de (Gordillo, 2019), (Chida, 2020), (Gascón , Jiménez, \& Pérez , 2015), (Palacios, 2018), (Allende, Evaluación geológico-ambiental en la determinación de la factibilidad de un área para relleno sanitario, 2001), (Allende, Estudio geológico-geotécnico del área para el relleno sanitario de la ciudad de Suyo, Provincia Ayabaca, Departamento de Piura, 2012), (Espejo, 2017), son los de permeabilidad, pendiente, precipitación, recursos hídricos y red vial; mientras que los criterios singulares fueron sitios arqueológicos, pasivos mineros, peligros geológicos, fallas geológicas y áreas nacionales protegidas.

El procedimiento trabajado en ArcGis concuerda con (Espejo, 2017) y (Javier \& Alcántara , 2018) donde elaboró mapas temáticos en formato raster por cada criterio utilizando las herramientas de ArcToolbox. En el proceso del análisis espacial multicriterio y el proceso de análisis jerárquico de los criterios (Saaty, 1977) se tomó como referencia al Manual para la Evaluación de Riesgos Originados por Fenómenos Naturales (CENEPRED, 2014) donde concuerda con la metodología de análisis y evaluación de riesgos.

Con los resultados del procedimiento se logró verificar que la ubicación actual del botadero Rumiallana (Figura 5) se encuentra en áreas No Aptas para la localización de relleno sanitario lo cual concuerda con lo verificado por la Contraloría (Contraloría General de la República, 2018).

\section{CONCLUSIONES}

- Se corrobora que los sistemas de información geográfica junto con el análisis espacial multicriterio y el análisis jerárquico son adecuados en la determinación de localización adecuada de rellenos sanitarios.

- Se obtuvieron seis áreas que serían adecuadas para la ubicación de rellenos sanitarios, con una evaluación complementaria especifica se tendrían más herramientas para descartar y obtener el área óptima.

- La metodología planteada en la presente investigación puede ser extrapolada a otros ámbitos, donde se pueden involucrar más criterios con el fin de enriquecer el procedimiento cartográfico.

\section{AGRADECIMIENTOS}

Agradezco a la unidad de posgrado de la facultad de Ingeniería Geológica, Minera, Metalúrgica y Geográfica de la Universidad Nacional Mayor de San Marcos, y al Instituto de Investigación de la FIGMMG por facilitar la publicación de la presente investigación.

\section{REFERENCIAS}

Allende, T. (2001). Evaluación geológico-ambiental en la determinación de la factibilidad de un área para relleno sanitario. Revista del Instituto de investigación de la Facultad de Minas, Metalurgia y Ciencias Geográficas, 
4(7), 52-62. Obtenido de https://revistasinvestigacion. unmsm.edu.pe/index.php/iigeo/article/view/2566

Allende, T. (2012). Estudio geológico-geotécnico del área para el relleno sanitario de la ciudad de Suyo, Provincia Ayabaca, Departamento de Piura. Revista del Instituto de investigación de la Facultad de Minas, Metalurgia y Ciencias Geográficas, 4(7), 52-62. Obtenido de https:// revistasinvestigacion.unmsm.edu.pe/index.php/iigeo/ article/view/721

Bahrani, S., Ebadi, T., Ehsani, H., Yousefi, H., \& Maknoon , R. (2016). Modeling landfill site selection by multi-criteria decision making and fuzzy functions in GIS, case study: Shabestar, Iran. Environmental Earth Sciences, Vol. 75, No. 4, pp. 337. Obtenido de https://link.springer.com/ article/10.1007/s12665-015-5146-4

Barredo, J., \& Gomez, M. (2005). Sistemas de Información Geográfica y Evaluación Multicriterio en la Ordenación del Territorio. España: Ra-Ma S.A. Editorial Y Publicaciones. Obtenido de https:/www.ra-ma.es/libro/sistemas-deinformacion-geografica-y-evaluacion-multicriterio-en-laordenacion-del-territorio-2a-ed_119356/

Çeliker, M., Yıldız, O., \& Nacar, N. (2019). Evaluating solid waste landfill site selection using multi-criteria decision analysis and geographic information systems in the city of Elazı̆g, Turkey. Pamukkale Univ Muh Bilim Derg, 25(6), 683-691. doi:10.5505/pajes.2018.70493

CENEPRED. (2014). Manual para la Evaluación de Riesgos Originados por Fenómenos Naturales. Obtenido de https:// www.cenepred.gob.pe/web/wp-content/uploads/Guia Manuales/Manual-Evaluacion-de-Riesgos_v2.pdf

Chida, K. (2020). Análisis multicriterio basado en sig para identificar potenciales áreas para establecer un relleno sanitario en el cantón Tena de la provincia Napo, Ecuador. Tesis de Ingeniería Ambiental, Universidad Estatal Amazónica, Ecuador. Obtenido de https://repositorio.uea. edu.ec/bitstream/123456789/810/1/T.AMB.B.UEA.\%20 $\% 203249 . p d f$

Contraloría General de la República. (2018). N N 422-2018CG: CONTRALORÍA ADVIRTIÓ RIESGOS EN EL BOTADERO "RUMIALLANA" EN PASCO. Obtenido de https://www.contraloria.gob.pe/wps/wcm/connect/ cgrnew/as_contraloria/prensa/notas_de_prensa/2018/pasco/ np $422-2018-c g$

Deswal, M., \& Laura, J. (2018). GIS based modeling using Analytic Hierarchy Process (AHP) for optimization of landfill site selection of Rohtak city, Haryana (India). Journal of Applied and natural Science 10(2):633-642. doi:https://doi.org/10.31018/jans.v10i2.1753

Ding, Z., Zhu, M., Wu, H., \& Zuo, J. (2020). Information system with multiple data layer approach to select the C\&D waste landfilling infrastructure. Environmental Science and Pollution Research 27:38788-38804. doi:https://doi. org/10.1007/s11356-020-09951-2

Espejo, A. (2017). Localización óptima de un relleno sanitario empleando sistemas de información geográfica distrito de Chachapoyas Amazonas. Tesis de Grado. Universidad Nacional Toribio Rodríguez de Mendoza. Obtenido de http://repositorio.unrtm.edu.pe/handle/UNTRM/1295
Gascón , S., Jiménez, L., \& Pérez, H. (2015). Óptima ubicación de un relleno sanitario para el Área Metropolitana del Valle de Aburrá empleando sistemas de información geográfica. Revista Ingenierías USBMed Vol. 6, $N^{o} .1$, págs. 38-45. Obtenido de https://dialnet.unirioja.es/servlet/ articulo? codigo $=6007720$

Gordillo, C. (2019). Localización de un relleno sanitario en el cantón Naranjal, mediante proceso de análisis jerárquico basado en sistemas de información geográfica. Tesis de grado. Universidad de Guayaquil, Ecuador. Obtenido de http://repositorio.ug.edu.ec/handle/redug/39601

Javier , L., \& Alcántara , F. (2018). Modelo cartográfico para determinar áreas óptimas para la expansión urbana del distrito de Huánuco como aporte para la planificación. Revista del Instituto de investigación de la Facultad de Minas, Metalurgia y Ciencias Geográficas, 21(42), 3-12. Obtenido de https://revistasinvestigacion.unmsm.edu.pe/ index.php/iigeo/article/view/15783

Ministerio del Ambiente. (2017). DS No 014-2017-MINAM "Reglamento del Decreto Legislativo $\mathrm{N}^{\circ}$ 1278, Decreto Legislativo que aprueba la Ley de Gestión Integral de Residuos Sólidos". Obtenido de https://www.minam.gob. pe/disposiciones/decreto-supremo-n-014-2017-minam/

Ministerio del Ambiente. (2018). RM No 459-2018-MINAM, Guía para el Diseño y Construcción de Infraestructuras de Disposición Final de Residuos Sólidos Municipales. Obtenido de https://www.gob.pe/institucion/minam/ normas-legales/237045-459-2018-minam

Ministerio del Ambiente. (2020). Reporte: Ancash: estadísticas ambientales, Junio 2020. Obtenido de https://sinia.minam. gob.pe/documentos/ancash-estadisticas-ambientalesdiciembre-2019

Ministerio del Ambiente. (2021). Listado de Rellenos Sanitarios del Perú. Obtenido de https://www.gob.pe/institucion/ minam/informes-publicaciones/279709-listado-de-rellenossanitarios

Mohammed, H., Majid, Z., Yamusa, Y., Mohd, M., Idris, K., \& Darwin, N. (2019). Sanitary Landfill Siting Using GIS and AHP: A Case Study in Johor Bahru, Malaysia. Engineering, Technology y Applied Science Research, Vol. $9 \mathrm{~N}^{\circ} 3,4100$ 4104. Obtenido de https://www.etasr.com/index.php/ ETASR/article/view/2633

Moreno-Jiménez, J., Aguarón, J., \& Escobar, M. (2001). Metodología científica en valoración y selección ambiental. Pesquisa Operacional Vol. 21, No. 1, p. 1-16. Obtenido de https://www.scielo.br/j/pope/a/ h8HCPW8rcJrxMs8cY8g4qDg/?format=pdf\&lang=es

Nguyen, X., Chou, T., Fang, Y., Hoang, T., \& Nguyen, Q. (2020). Optimal Position Proposal for Construction of Municipal Solid Waste Landfill Using an Approach of Fuzzy Set Theory and AHP in a GIS Environment: A Case Study in Hoai Duc District, Hanoi City, Vietnam. International Journal of Geoinformatics, Volume 16, No. 2. Online ISSN 2673-0014. Obtenido de https://journals.sfu.ca/ijg/index. php/journal/article/view/1811

Organismo de Evaluación y Fiscalización Ambiental (2021). Inventario Nacional de Áreas Degradadas por Residuos Sólidos Municipales. Perú. Obtenido de https://oefa.maps.arcgis.com/apps/webappviewer/index. html?id=6a530906bcdd44d388d6c032d7cb844a 
Palacios, I. (2018). Evaluación multicriterio para la ubicación de un relleno sanitario en la ciudad de Macas, a través de la ponderación de sus variables con el proceso analítico jerárquico, ahp. Revista de Ciencias de Seguridad y Defensa, Vol. III, No 3. Obtenido de http://geol.espe.edu.ec/ wp-content/uploads/2018/06/7.pdf

Posada, H. (2015). Evaluación multicriterio y SIG., como herramientas para la gestión territorial (Caso de estudio ubicación del terminal de transporte en Zipaquirá Cundinamarca). (Tesis de grado). Universidad Santo Tomás, Colombia. Obtenido de https://hdl.handle.net/11634/2270
Saaty, T. (1977). A scaling method for priorities in hierarchical structures. Journal of Mathematical Psychology. Obtenido de https://www.sciencedirect.com/science/article/abs/ pii/0022249677900335?via\%3Dihub

Sisay, G., Legesse, S., \& Getahun, K. (2020). GIS-based potential landfill site selection using MCDM-AHP modeling of Gondar Town, Ethiopia. African Geographical Review. Routledge Taylor \& Franzcis Group Volume 40. doi:https:// doi.org/10.1080/19376812.2020.1770105 